Relations industrielles

Industrial Relations

\title{
Stress, santé et rendement au travail, par Shimon Dolan et André Arsenault, Monographie no 5, Montréal, École des relations industrielles de l'Université de Montréal, 1980, 186 pp.
}

\section{René Boulard}

Volume 36, numéro 3, 1981

URI : https://id.erudit.org/iderudit/029196ar

DOI : https://doi.org/10.7202/029196ar

Aller au sommaire du numéro

Éditeur(s)

Département des relations industrielles de l'Université Laval

ISSN

0034-379X (imprimé)

1703-8138 (numérique)

Découvrir la revue

Citer ce compte rendu

Boulard, R. (1981). Compte rendu de [Stress, santé et rendement au travail, par Shimon Dolan et André Arsenault, Monographie no 5, Montréal, École des relations industrielles de l'Université de Montréal, 1980, 186 pp.] Relations industrielles / Industrial Relations, 36(3), 701-702.

https://doi.org/10.7202/029196ar

Tous droits réservés @ Département des relations industrielles de l'Université Laval, 1981
Ce document est protégé par la loi sur le droit d'auteur. L'utilisation des services d’Érudit (y compris la reproduction) est assujettie à sa politique d'utilisation que vous pouvez consulter en ligne.

https://apropos.erudit.org/fr/usagers/politique-dutilisation/ 
Enfin, si Mirella Giannini révèle par certaines affirmations, sa nette préférence pour les comportements de type «déviant» des magistrats, cela ne la prive pas d'objectivité dans la présentation et l'interprétation des résultats de sa recherche.

\section{Danielle PICARD}

Université de Paris I Panthéon-Sorbonne

Stress, santé et rendement au travail, par Shimon Dolan et André Arsenault, Monographie no 5, Montréal, École des relations industrielles de l'Université de Montréal, 1980, 186 pp.

Pour tous ceux qui s'intéressent aux problèmes de santé et de sécurité au travail, la monographie de Dolan et Arsenault représente un ouvrage attendu depuis longtemps. En effet, cette monographie présente l'avantage énorme de s'attaquer à un problème extrêmement complexe, celui du stress au travail, et de réussir à placer simplement chaque morceau du casse-tête pour en dégager une image passablement claire.

Après avoir parlé de "santé du milieu de travail» (chapitre 1), les auteurs consacrent deux chapitres au concept de qualité de vie au travail (sous l'angle de la satisfaction au travail, puis sous celui du stress), un autre au concept de stress, deux autres aux «origines et conséquences» du stress au travail, pour déboucher, au chapitre 7 sur «une approche multidisciplinaire à la notion de stress au travail». Le dernier chapitre fait état d'un «modèle clinique d'intervention en milieu du travail».

L'ouvrage est le résultat d'une collaboration étonnante: "Que le lecteur surpris par le fait qu'un docteur en psychologie organisationnelle et un médecin spécialiste en médecine nucléaire aient réussi à s'entendre et à se comprendre suffisamment pour en arriver à écrire une monographie sur le stress au travail se rassure: nous sommes surpris nousmêmes» (Introduction, p. 17). Le résultat est tout aussi étonnant que la collaboration.
En effet, il s'agit là d'une des meilleures synthèses sur le stress au travail. Les différentes dimensions des paramètres en présence (santé, satisfaction, stress) et les nombreuses définitions qui en ont été données sont clairement évoquées. Ce qui plus est, les liens entre eux sont établis. Pour y arriver, les auteurs se placent au-dessus des controverses habituelles (approche organiciste - approche psychologique; etc.) et élaborent, pièce par pièce, les éléments de leurs synthèse. Celle-ci s'articule autour des besoins tant organisationnels qu'individuels, à court, moyen et long terme, et des liens entre ces différentes composantes. Si l'ouvrage en arrive à un résultat intéressant, c'est que les auteurs ont su partir de plus loin, prendre du recul par rapport au stress lui-même, et pousser l'analyse autant que faire se peut en intégrant les différents éléments rencontrés.

Le déroulement logique de leur argumentation (si tant est qu'il y ait argumentation!) est relativement facile à suivre. "Chaque chapitre comporte une infrastructure où nous présentons dans un premier temps les thèses générales soutenues dont nous prenons systématiquement le contre-pied pour enfin tenter de dégager une synthèse qui corresponde à notre interprétation» (p. 18). Chaque chapitre, et chaque partie de chapitre, présente d'ailleurs une excellente synthèse. De même, les différents tableaux, présentés souvent selon une même typologie, permettent au lecteur de s'y retrouver facilement.

Il y a beaucoup de rigueur dans la présentation et l'interprétation des définitions de concepts souvent flous et difficiles, et des différents modèles couramment utilisés, entre autres, en psychologie sociale et organisationnelle. Le concept de stress lui-même est analysé dans toutes ses dimensions, ses antécédents et conséquents, toute cette analyse débouchant sur un modèle conceptuel (que les auteurs appellent «modèle conditionnel du stress au travail» - p. 143). Le cadre général de ce modèle, de même que plusieurs de ses composantes, est emprunté à Cooper et Marshall (Journal of Occupational Psychology, 1976, vol. 49, pp. 11-28). Les auteurs 
semblent plutôt en faire remonter la source à un autre de leurs ouvrages de 1979.

En conclusion, la monographie de Dolan et Arsenault représente un apport précieux à l'étude du stress au travail. En étudiant un phénomène intrinsèquement conflictuel (soit le stress défini comme une incapacité, perçue comme dommageable, de l'individu à faire face à une force de l'environnement), les auteurs ont un parti pris très évident de réconcilier les objectifs organisationnels et les objectifs individuels. Ce faisant, ils présentent une synthèse très fructueuse du phénomène à l'étude et un aperçu intéressant des interventions possibles:

«On peut dire que cette nouvelle perspective est à la croisée de trois disciplines traditionnelles: le management, la psychologie et la médecine... Il ne s'agit pas d'éliminer le stress, mais de le «gérer» et de l'ajuster à un niveau de concordance optimal individuemploi, promouvant ainsi à la fois le bienêtre individuel et le bien-être organisationnel.» (p. 176).

Si tel était l'objectif, la façon d'aborder le problème dans l'ouvrage permet de constater que les auteurs ont fait avancer suffisamment la question pour penser qu'un pas de plus a été franchi dans cette direction.

René BOULARD

Université Laval

Perdre sa vie à la gagner; manuel pour la santé des travailleurs, par Jeanne $M$. Stellman et Susan M. Daum; version française de "Work is Dangerous to your Health", traduit par Louise E. Lavoie et Zita De Koninck; Montréal, Édition Parti pris, 1979, 493 pp.

Comme la seconde partie du titre le mentionne, le présent ouvrage se veut un «manuel pour la santé des travailleurs». L'à-propos et l'utilité d'un tel ouvrage-synthèse ne peuvent être mis en cause, du moins théoriquement.

Le bouquin se présente comme une version plus abordable (il s'agit d'un ouvrage de vulgarisation destiné d'abord aux travailleurs) que les «bibles» connues dans le domaine (Sax, Hunter, Scherrer, etc.). En effet, chacun des chapitres constitue une bonne synthèse, en général assez bien faite et relativement exhaustive. Après avoir posé le problème des maladies industrielles en général (chap. 1), rappelé les grandes lignes de l'anatomie et surtout de la physiologie du corps humain (chap. 2), les auteurs abordent un par un, les différents dangers liés au travail: stress (chap. 3), bruit et vibrations (chap. 4), chaleur et froid (chap. 5), lumière, rayon-X, radiation (chap. 6), dangers chimiques (chap. 7), dangers du soudage (chap. 8), contrôle de la pollution au travail (chap. 9) et dans l'environnement (chap. 10). Les deux derniers chapitres parlent de la conservation des dossiers de santé et des autres moyens d'action.

Il s'agit d'un livre de référence qui, à plusieurs points de vue, peut s'avérer fort utile, et dont plusieurs revendications peuvent trouver des échos au Québec. Il en est ainsi des parties sur l'amiantose, sur l'inadéquacité des normes gouvernementales (v.g. $90 \mathrm{db} \mathrm{A}$ ), sur leur non-respect par l'entreprise, sur le camouflage des accidents, etc.

L'ouvrage de Stellman et Daum fait preuve d'un penchant évident pro-travailleur, pro-syndicat et anti-employeur. Ce penchant est fort louable en soi, mais, à l'occasion, l'objectivité des comptes rendus en est légèrement affectée. Des hypothèses pour les chercheurs apparaissent comme des certitudes dans le volume; les sources ne sont pas toujours identifiées de sorte que certains énoncés paraissent des affirmations gratuites; on insiste beaucoup sur le rôle du syndicat, mais les non-syndiqués ne se voient offrir d'autre choix que de se syndiquer.

On dénote également une teinte un peu alarmiste. Il est vrai que si un agent agresseur est réputé néfaste, il ne devrait pas être traité à la légère, mais l'élimination de certains agresseurs est parfois très complexe et leur remplacement impossible, à court terme; aussi est-il préférable de connaître à. fond le problème auquel on est confronté. Une attitude «scientifique» peut probablement faire au- 